\title{
Visible-Light-Induced Photocatalytic Activity of Stacked MXene
}

\section{Sheets of $\mathrm{Y}_{2} \mathrm{CF}_{2}$}

\author{
Kazuhiko Maeda, ${ }^{1 *}$ Haruki Wakayama, ${ }^{1}$ Yasuhito Washio, ${ }^{2}$ Asako Ishikawa, ${ }^{2}$ Megumi Okazaki, ${ }^{1}$ \\ Hiroko Nakata, ${ }^{1}$ Satoru Matsuishi ${ }^{2 *}$
}

${ }^{1}$ Department of Chemistry, School of Science, Tokyo Institute of Technology, 2-12-1-NE-2 Ookayama, Meguro-ku, Tokyo 152-8550, Japan

2 Materials Research Center for Element Strategy, Tokyo Institute of Technology, 4259-SE-2 Nagatsuta-cho, Midori-ku, Yokohama 226-8503, Japan

*To whom corresponding authors should be addressed.

K.M.: maedak@chem.titech.ac.jp; S.M.: matsuishi@mces.titech.ac.jp

\section{Table of Contents}

Table S1. Refinement of powder XRD pattern of the as-synthesized $\mathrm{Y}_{2} \mathrm{CF}_{2}$ sample $\quad$...S2

Table S2. Surface atomic ratios of $\mathrm{Y}_{2} \mathrm{CF}_{2}$ before and after the photoreaction $\quad$...S2

Figure S1. Time course of $\mathrm{H}_{2}$ evolution using $\mathrm{Y}_{2} \mathrm{CF}_{2}$ under visible light $(\lambda>700 \mathrm{~nm}) \quad \ldots \mathrm{S} 3$

Figure S2. Characterization of $\mathrm{Y}_{2} \mathrm{CF}_{2}$ before and after photoreaction $\quad . . \mathrm{S} 3$

Additional Notes on Reaction Solvent $\quad$...S4

References $\quad$...S4 
Table S1. Refinement of powder XRD pattern of the as-synthesized $\mathrm{Y}_{2} \mathrm{CF}_{2}$ sample.

\begin{tabular}{|c|c|c|c|c|}
\hline Refined parameter & $\mathrm{Y}_{2} \mathrm{CF}_{2}(P \overline{3} m 1)$ & \multicolumn{2}{|c|}{$\mathrm{YF}_{3}(P n m a)$} & YOF $(P 4 / n m m)$ \\
\hline Phase content / wt.\% & $94.7(5)$ & \multicolumn{2}{|l|}{$2.8(4)$} & $2.5(2)$ \\
\hline$a / \AA$ & $3.66760(5)$ & \multicolumn{2}{|l|}{$6.374(8)$} & $3.91543(5)$ \\
\hline$b / \AA$ & $=a$ & \multicolumn{2}{|l|}{$6.847(7)$} & $=a$ \\
\hline$c / \AA$ & $6.3054(1)$ & \multicolumn{2}{|l|}{$4.219(5)$} & $5.407(1)$ \\
\hline \multicolumn{2}{|l|}{$\mathrm{Y} 1,2 d(0,0, z)$} & \multicolumn{2}{|c|}{$\mathrm{Y} 1,4 c(x, 1 / 4, z)$} & $\mathrm{Y} 1,2 c(1 / 2,0, z)$ \\
\hline$z$ & $0.2163(2)$ & $x$ & 0.3673 & $\begin{array}{ll}z & 0.2118\end{array}$ \\
\hline$B_{\mathrm{eq}} / \AA^{2}$ & $0.55(5)$ & $z$ & 0.0613 & $B_{\text {eq }} / \AA^{2}$ \\
\hline \multicolumn{2}{|l|}{$\mathrm{C} 1,1 a,(0,0,0)$} & $B_{\text {eq }} / \AA^{2}$ & 0.23 & $\mathrm{~F} 1,2 a,(0,0,0)$ \\
\hline$B_{\text {eq }} / \AA^{2}$ & $1.9(8)$ & \multicolumn{2}{|c|}{$\mathrm{F} 1,4 c(x, 1 / 4, z)$} & $B_{\text {eq }} / \AA^{2} \quad 1.0$ \\
\hline \multicolumn{2}{|l|}{$\mathrm{F} 1,2 d,(0,0, z)$} & $x$ & 0.5355 & $\mathrm{O} 1,2 b,(0,0,1 / 2)$ \\
\hline$z$ & $0.6211(9)$ & $z$ & 0.5952 & $B_{\mathrm{eq}} / \AA^{2}$ \\
\hline \multirow[t]{6}{*}{$B_{\text {eq }} / \AA^{2}$} & $1.7(2)$ & $B_{\mathrm{eq}} / \AA^{2}$ & 0.59 & \\
\hline & & \multicolumn{2}{|c|}{$\mathrm{F} 2,8 d(x, \mathrm{y}, z)$} & \\
\hline & & $x$ & 0.1610 & \\
\hline & & $y$ & 0.0638 & \\
\hline & & $z$ & 0.3772 & \\
\hline & & $B_{\text {eq }} / \AA^{2}$ & 0.67 & \\
\hline$R_{\exp }$ & $3.35 \%$ & & & \\
\hline$R_{\mathrm{wp}}$ & $4.37 \%$ & & & \\
\hline$R_{\mathrm{p}}$ & $3.79 \%$ & & & \\
\hline$\chi^{2}$ & 1.41 & & & \\
\hline
\end{tabular}

Table S2. Surface atomic ratios of $\mathrm{Y}_{2} \mathrm{CF}_{2}$ before and after the photoreaction (20-h of visible light irradiation, Figure 3B). The original XPS data are shown in Figure 4A.

\begin{tabular}{cccc}
\hline Sample & \multicolumn{3}{c}{ Surface atomic ratios } \\
\cline { 2 - 4 } & $\mathrm{C} / \mathrm{Y}$ & $\mathrm{F} / \mathrm{Y}$ & $\mathrm{O} / \mathrm{Y}$ \\
\hline Before & 2.9 & 0.45 & 2.4 \\
After & 4.8 & 1.3 & 2.9 \\
\hline
\end{tabular}




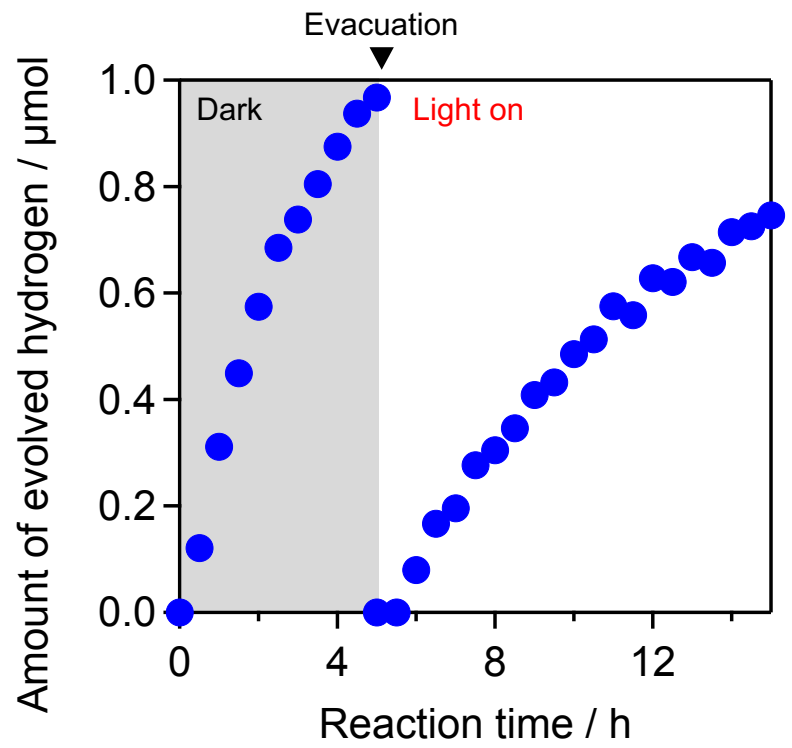

Figure S1. Time course of $\mathrm{H}_{2}$ evolution using $\mathrm{Y}_{2} \mathrm{CF}_{2}$ under visible light $(\lambda>700 \mathrm{~nm})$. Reaction conditions: catalyst, $50 \mathrm{mg}\left(0.2 \mathrm{wt} \% \mathrm{Pt}\right.$ in situ photodeposition from $\left.\mathrm{H}_{2} \mathrm{PtCl}_{6} \cdot 6 \mathrm{H}_{2} \mathrm{O}\right)$; reactant solution, a mixed solution of MeCN and TEOA (13:1 v/v), $140 \mathrm{~mL}$; light source, Xe lamp (300 W) with a CM-1 cold mirror and cutoff filters (L42 + R70). Output current of the Xe lamp was $10 \mathrm{~A}$.

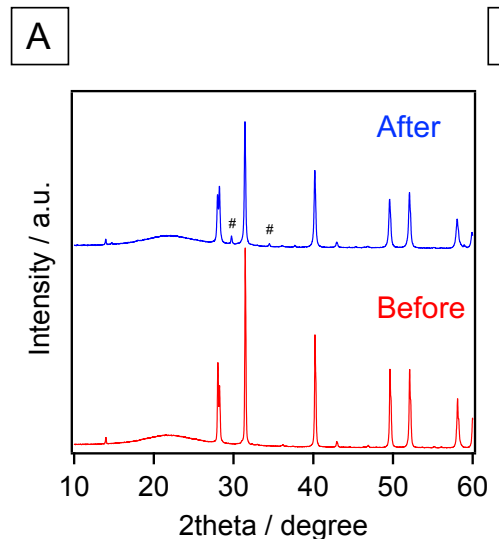

$\mathrm{B}$
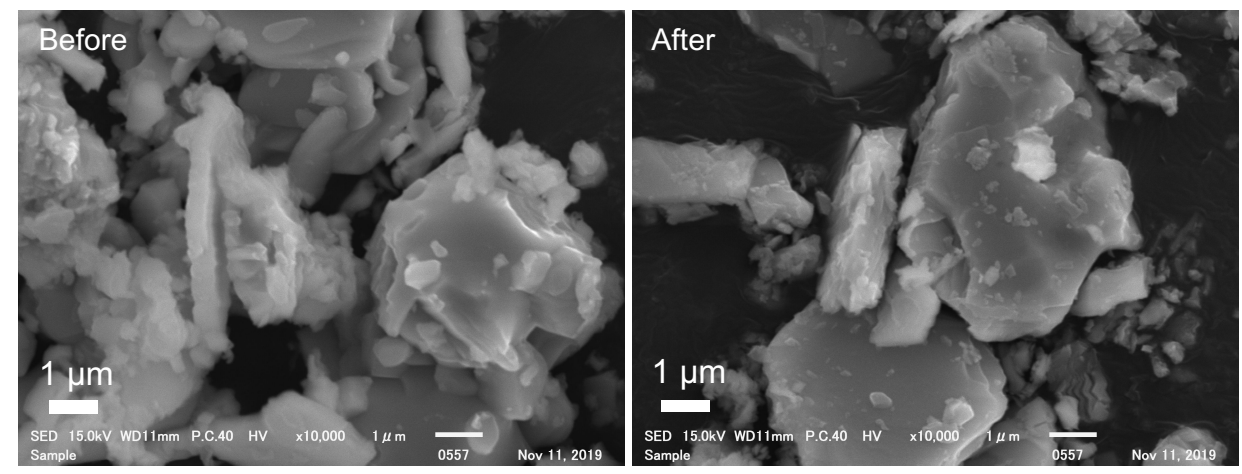

Figure S2. (A) XRD patterns and (B) SEM images of $\mathrm{Y}_{2} \mathrm{CF}_{2}$ before and after photoreaction (20-h of visible light irradiation, Figure $3 \mathrm{~B}$ ). Although small unknown peaks (marked with \#) were observed, the original crystal structure of $\mathrm{Y}_{2} \mathrm{CF}_{2}$ was mostly kept, as seen in the panel $\mathrm{B}$. 


\section{Additional Notes on Reaction Solvent}

Another candidate of organic solvent is $N, N$-dimethylacetamide (DMA), which is (photo)chemically stable. ${ }^{\mathrm{S} 1}$ However, our previous study on photocatalytic $\mathrm{H}_{2}$ evolution using Pt-loaded mesoporous graphitic $\mathrm{C}_{3} \mathrm{~N}_{4}$ from solvent/TEOA mixtures indicated that DMA was rather bad solvent, while MeCN gave relatively high activity. ${ }^{\mathrm{S} 2}$ Therefore, the use of MeCN as the main solvent in this work should be reasonable.

\section{References}

S1. Kuramochi, Y.; Kamiya, M.; Ishida, H. Photocatalytic $\mathrm{CO}_{2}$ Reduction in N,N-Dimethylacetamide/Water as an Alternative Solvent System. Inorg. Chem. 2014, 53, 3326-3332.

S2. $\quad$ Kuriki, R.; Sekizawa, K.; Ishitani, O.; Maeda, K. Visible-Light-Driven $\mathrm{CO}_{2}$ Reduction with Carbon Nitride: Enhancing the Activity of Ruthenium Catalysts. Angew. Chem. Int. Ed. 2015, 54, 2406-2409. 